\title{
Optimal Resource Scheduling for laaS Cloud Computing using Cuckoo Search Algorithm
}

\author{
Syed Hamid Hussain Madni ${ }^{a}$, Muhammad Shafie Abd Latiff ${ }^{*}$, Shafi'i Muhammad Abdulhamida,b \\ ${ }^{a}$ Faculty of Computing, University Teknologi Malaysia, 81310 UTM Johor Bahru, Johor, Malaysia \\ ${ }^{b}$ Department of Cyber Security Science, Federal University of Technology Minna, Nigeria
}

*Corresponding author: shafie@utm.my

\begin{abstract}
Effective resource scheduling is essential for the overall performance of cloud computing system. Resource scheduling problem in IaaS cloud computing is investigated in this paper. It is established to be an NP-hard problem. A recently developed Cuckoo Search (CS) meta-heuristic algorithm is proposed in this paper, to minimize the response time, makespan and throughput for the resource scheduling in IaaS cloud computing. Simulation results show that CS algorithm outperforms that of Ant Colony Optimization (ACO) algorithm based on the considered parameters.
\end{abstract}

Keywords: Resource scheduling; IaaS; meta-heuristic; cuckoo search; cloud computing

(C) 2017 Penerbit UTM Press. All rights reserved

\subsection{INTRODUCTION}

Cloud technology is recently used everywhere and it is a utility service that gives access to remote resources managed by experts which is available on-demand. Simply these services are managed over the internet with no upfront cost and pay only for the usage time (Foster et al., 2008, Mell and Grance, 2009). In cloud computing, for the effective performance of any system, there is a need for effective scheduling. It is basically a procedure of assigning various tasks to given available resources within a feasible period of time. Through this, different types of tasks are scheduled to different types of virtual resources by considering some constraints given by cloud providers and users. The constraints on the side of cloud users that are deadline or budget, while from the cloud provider's side are maximizing the revenue, profit and utilization (Abdulhamid et al., 2014, Madni et al., 2016a).

Resource is something that is requisite to carry out a process in the cloud. For example, processor for data processing and data storage devices or network connection for data transporting (Armbrust et al., 2010, Madni et al., 2016b). Task represents the computation unit run on a node and it is considered to be an indivisible schedulable unit. Whereas a job is a computational activity made up of various tasks that have need of numerous processing competencies and diverse essential (CPU, Nodes, Storage, Speed, etc.) and constraints, frequently expressed within job description. Each job requires various parameters such as required data, execution time, makespan, throughput, priority, etc (Abdulhamid and Latiff, 2014, Achar et al., 2012).

Optimization is a procedure of examining for the ideal solution to a specific problem of attention. Optimization algorithms are advanced techniques for solving an optimization problem with an objective in order to find out its optimality, though such optimality is not constantly within reach. An optimization criterion is used, when scheduling decisions are made and represent the goals of the scheduling process. The objective function is decided for designing scheduling process. Mathematically, an algorithm is a technique to produce outputs for a given sets of inputs. From optimization point of view, an optimization algorithm generates a new solution $\mathrm{x}^{\mathrm{t}+1}$ to a given problem from an identified solution $\mathrm{x}^{\mathrm{t}}$ at time $t$ or iteration.

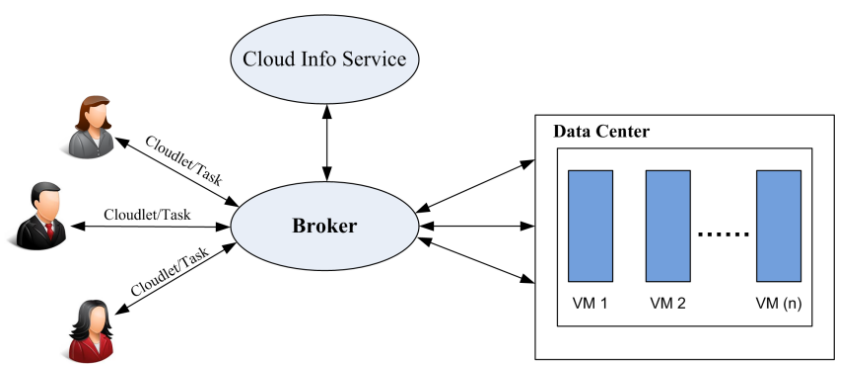

Figure 1 Resource scheduling for IaaS in cloud computing 
Resource scheduling allocates the specific and accurate cloudlet or task to the cloud users in cloud computing. The intention behind this is the extreme usage of resources, as illustrated in Figure 1. However, well-ordered and efficient scheduling is the obligatory for both cloud providers cloud users (Madni et al., 2016a). The most challenging problem in resource scheduling for IaaS in cloud computing is handling and providing efficient utilization of resources (Manvi and Krishna Shyam, 2014). Our work aims to describe a proficient resource scheduling algorithm for cloud computing, which efficiently manages the resources based on the some quality metrics.

In this paper, we propose an optimal CS meta-heuristic population based algorithm for resource scheduling in IaaS cloud computing. The parameters used for determining the performance of scheduling algorithms in the simulation are based on three factors: Response Time, Makespan and throughput. The proposed technique is also capered with familiarized meta-heuristic technique for resource scheduling in IaaS cloud computing.

The remainder of this paper is structured as follows. In the next section, we review the background studies of CS algorithm in cloud computing and other areas, also review the current studies of ACO for resource scheduling in cloud computing. We provide the description of CS optimization algorithm in the methodology section. Results and discussion show the performance evaluation of CS algorithm with the help of experimental simulation. The last section focuses on the conclusion and future works.

\subsection{BACKGROUND}

This section reviews the application of Cuckoo Search by previous studies. Based on the reviewed literatures, the major areas that used Cuckoo Search to solve optimization problems consist of data generation, data fusion, engineering, job scheduling, networking, pattern recognition, software testing and wireless sensor networks. In summary, from the reviews, it shows that CS is very efficient in solving many complex optimization problems.

Yang and Deb (2010) sort out several optimization problems using the CS algorithm. Yang and Deb (2013) also propose a multiobjective CS algorithm for design optimization problems. Gandomi et al. (2013) address the structural optimization problems using CS algorithm. Zendaoui and Layeb (2016) adapt the CS algorithm to resolve a one-dimensional bin packing problem. Dasgupta and Das (2015) and Wang et al. (2016) avail CS algorithm to solve the flow shop scheduling problem.

To solve the issue of optimal production planning Unit Commitment (UC), a CS algorithm is applied by (Gharegozi and Jahani, 2013). Discrete Cuckoo Search (DCS) is functioned to solve resource constrained project scheduling problem (Bibiks et al., 2015) and travelling salesman problem (Ouaarab et al., 2014). CS based method is evolved for scheduling optimization of a flexible manufacturing system. Furthermore, CS algorithm is applied for feature selection in cancer categorization by applying microarray gene expression data (Gunavathi and Premalatha, 2015).

Babukarthik et al. (2013) propose a hybrid algorithm, which combines the advantage of ACO and CS to minimize the makespan for cloud computing. In addition, Sait et al. (2016) apply the CS algorithm to solve the virtual machine placement problem of data centers in cloud computing. Xu et al. (2014) use CS algorithm to the analysis of big data by using MapReduce paradigm in cloud computing. Moreover, to enhance the internal utilization and reduce the price of outsourced tasks to the external cloud is determined using Cuckoo Search driven Particle Swarm Optimization (CS-PSO) method (Raju et al., 2016).

ACO algorithm is applied to discover the optimal resource allocation by minimizing the makespan in the dynamic cloud by Tawfeek et al. (2013). This technique with multiple objective optimizations including the cost, makespan, time deadline and utilization.(Zuo et al., 2015). ACO is also used to address resource scheduling problem in cloud regarding the real QoS parameters by increasing the resource utilization and minimizing the processing time (Zhu and Liang, 2013).

CS algorithm is considered as a successful technique due to the smaller number of iteration and convergence rate. It's dependencies to input data are decreased cause of its smaller number of calculations and input data. By adding Levy flight function, global search is conducted at the same time as local search in CS algorithm. That is why it is extensively rewarded attention by researchers to get the optimal results in different areas.

\subsection{METHODOLOGY}

Cuckoo search (CS) algorithm is an innovative nature-inspired meta-heuristic algorithm established by Yang and Deb. It is inspired by the obligate brood parasitic behaviour of few cuckoo species in a grouping with the Levy flight behaviour of certain birds and fruit flies in nature (Yang and Deb, 2009). CS algorithm is mostly used single parameter and famous due to its simplicity and easy to implement. The main objective is to speed up the convergence rate of the algorithm. It deals with multi-criteria optimization problems and still hybridized with other swarm based algorithms. Figure 2 displays the flow chart of the CS optimization algorithm. The CS algorithm is based on main three points are listed below.

- $\quad$ Each cuckoo lays one egg at a time and dumps it in a randomly chosen nest.

Egg $=$ nest + solution

- The best nest with the high quality of eggs (solution) will carry over to the next generation.

Best solution $=\min f(x)$ where $f(x)$ is an objective function

- The numbers of accessible host nests are static and a host can realize an unfamiliar egg with probability $(0,1)$.

Three parameters are used in CS algorithm

- $\quad P \alpha \in[0,1]$

Probability of worse nest to be abandoned

- $\alpha>0$

Step size, which should be related to the scale of the problem of interest. In most cases $\alpha>1$. 
- $\lambda$ step length random.

\section{Steps of CS Algorithms}

Step 1: Generate an initial population of $\mathrm{x}$ host nests.

$\mathrm{X}_{\mathrm{i}}=(\mathrm{i}=1,2,3, \ldots \ldots, \mathrm{n})$.

$\left(a_{i}, r_{i}\right)$ a candidate for optimal parameters

Step 2: Lay the egg $\left(a_{k^{\prime}}, \mathrm{r}^{\prime}\right)$ in the k nest. K nest is randomly selected. Cuckoo's egg is precise similar to host egg. where

- $\quad a_{k^{\prime}}=a_{k}+$ Randomwalk(Levy flight) $a_{k}$

- $\quad r_{k}^{\prime}=r_{k}+$ Randomwalk(Levy flight) $r_{k}$

Step 3: Compare the fitness of cuckoo's egg with the fitness of the host egg.

Choose a nest among $\mathrm{n}$ (say j) randomly

Step 4: If the fitness of cuckoo's egg is superior to host egg, substitute the egg in nest k by cuckoo's egg.

$$
\begin{gathered}
\text { if }(\mathrm{Fi}>\mathrm{Fj}) \\
\mathrm{X}_{\mathrm{i}}^{\mathrm{t}}=\mathrm{X}_{\mathrm{i}}^{\mathrm{t}+1} \\
\text { else } \\
\quad \mathrm{X}_{\mathrm{i}}^{\mathrm{t}+1}=\mathrm{x}_{\mathrm{i}}^{\mathrm{t}}
\end{gathered}
$$

Step 5: If host bird notices it, the nest is abandoned and new one is built ( $\mathrm{p}<0.25)$ (to avoid local optimization).

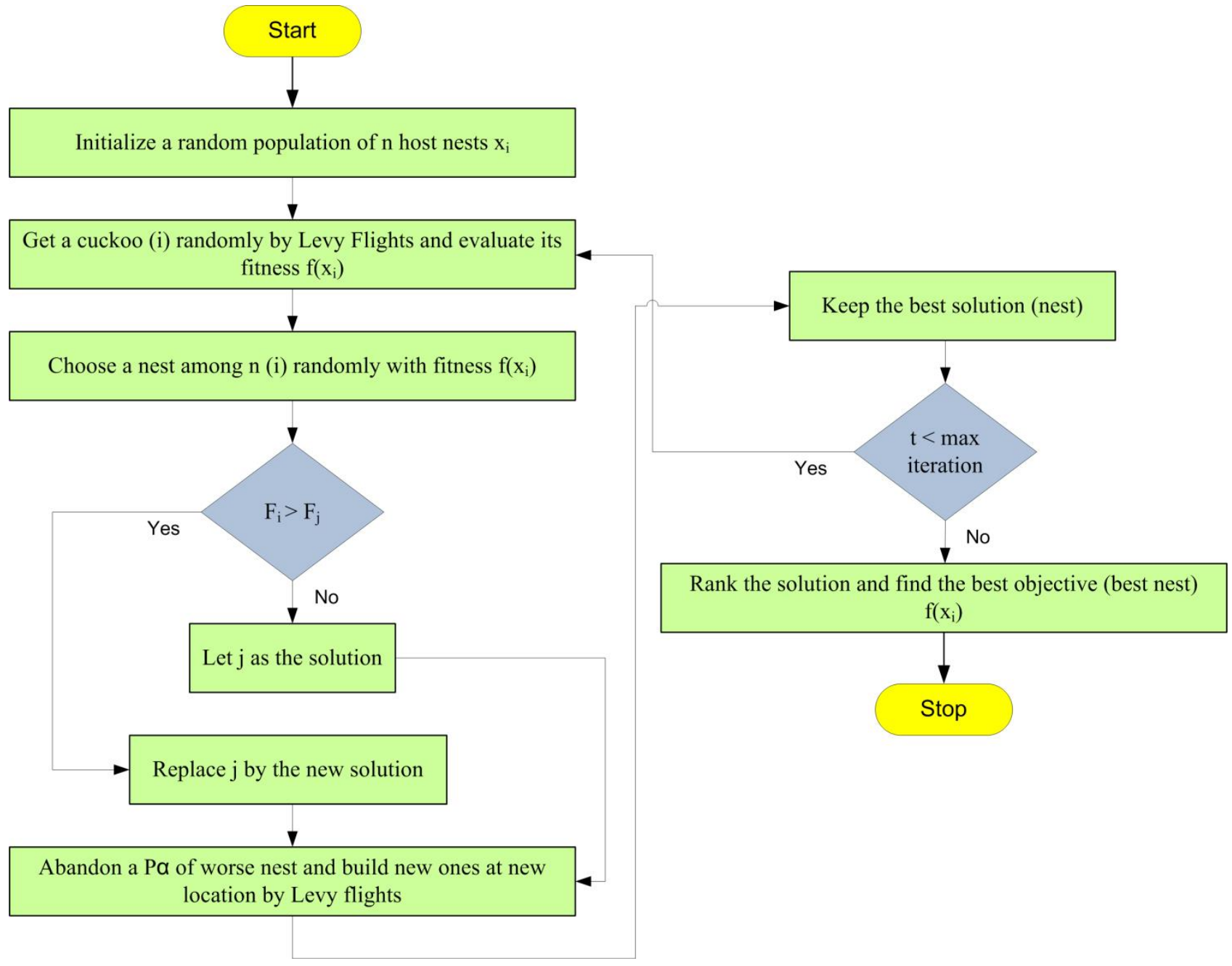

Figure 2 Flow chart of cuckoo search algorithm

Levy flight is a random walk in which the steps are defined in terms of the step length, which are distributed according to a heavy tailed probability distribution with the direction of steps being isotropic and random. 


$$
x_{i}^{t}=x_{i}^{t+1}+\alpha \oplus \operatorname{levy}(\lambda)
$$

where

$x_{i}^{t}$ new solution

$x_{i}^{t+1}$ current solution

$\alpha \bigoplus \operatorname{levy}(\lambda)$ transaction probability

Random walk is a Markov chain, whose next status/location only depends upon the current location and transaction probability. $\bigoplus$ is entrywise multiplication, it is similar to a product which is used by PSO algorithm.

\subsection{RESULTS AND DISCUSSIONS}

In this research, CloudSim is used to execute the CS optimization algorithm for the optimal resource scheduling in cloud computing. The experimental parameters setting of CloudSim is shown in Table 1. A comparative study has been done to estimate the performance of the recommended CS algorithm with respect to the existed ACO algorithm, with the ACO parameters set according to Tawfeek et al. (2013). This simulation mainly validates the advantage of CS algorithm with respect to the response time, makespan and throughput among these scheduling algorithms in the cloud computing environment. VMs are considered as resources in this research. We also measured the quality metrics with CS and ACO algorithms, while the number of VMs is fixed and cloudlets size are varied in the simulation. The simulation results are illustrated in Figure 3, 4 and 5.

Table 1 Experimental parameters setting of CloudSim

\begin{tabular}{|c|c|c|c|}
\hline Sr. No & Entity Type & Parameters & Values \\
\hline 1 & User & No of users & 5 \\
\hline \multirow{2}{*}{2} & \multirow{2}{*}{ Cloudlet } & No of cloudlets & 10 \\
\cline { 3 - 4 } & & Length & $1000-10000$ \\
\hline \multirow{3}{*}{3} & Host & No of Host & 2 \\
\cline { 3 - 4 } & & RAM & $512 \mathrm{MB}$ \\
\cline { 3 - 4 } & & Storage & 10000 \\
\cline { 3 - 4 } & & Bandwidth & 1000 \\
\hline \multirow{4}{*}{4} & \multirow{4}{*}{ Virtual Machine } & No of VMs & 4 \\
\cline { 3 - 4 } & & Type of Policy & Time Share \\
\cline { 3 - 4 } & & RAM & $512 \mathrm{MB}$ \\
\cline { 3 - 4 } & & Size & 1000 \\
\cline { 3 - 4 } & & VMM & Xen \\
\cline { 3 - 4 } & & Operating System & Linux \\
\cline { 3 - 4 } & & No of CPUs & 2 \\
\hline \multirow{2}{*}{5} & Data Center & No of Data Centers & 2 \\
\hline
\end{tabular}

\section{Response Time}

The response time refers to the summation of the submission time and waiting time. Precisely, response time is the time of task or cloudlet that it takes to respond to a specified input (Bashir et al., 2013).

$$
\operatorname{Res}_{\text {Time }}=\operatorname{task}_{i}\left(S b_{\text {Time }}+\operatorname{Str}_{\text {Time }}\right)
$$

where $S b_{\text {Time }}$ denotes the submission time and $S t r_{\text {Time }}$ represents the starting time of the cloudlet or task $i$.

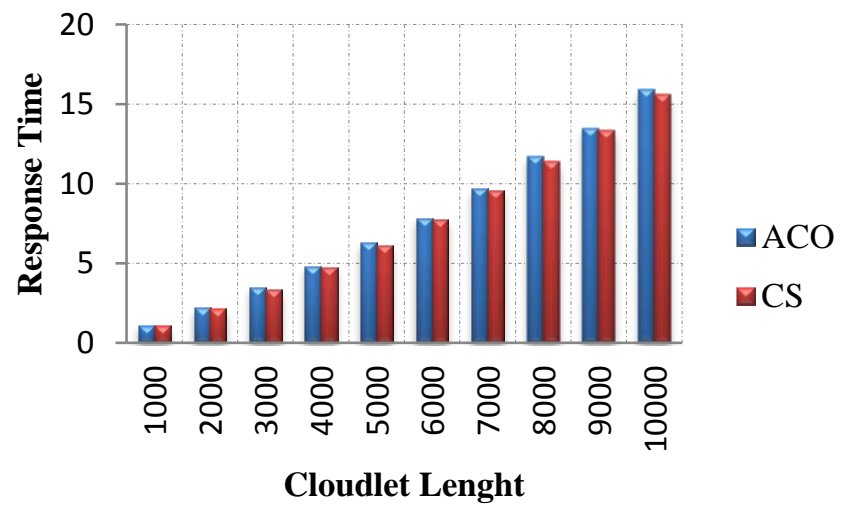

Figure 3 Comparison of response time for resource scheduling 
The results are presented in Figure 3 reflect that the response time CS algorithm is shorter than ACO algorithm. So CS optimization algorithm has higher efficiency for resource scheduling in cloud computing.

\section{Makespan}

Makespan identifies the maximum completion time of task or time when the resources are assigned to the users. If the makespan of specific cloudlet or task is not minimized then the demand will not be completed on time (Abdulhamid et al., 2015, Abdulhamid et al., 2016).

$$
\text { Makespan }=\max _{\text {task }^{i}}\left(\text { Fnh }_{\text {Time }}\right)
$$

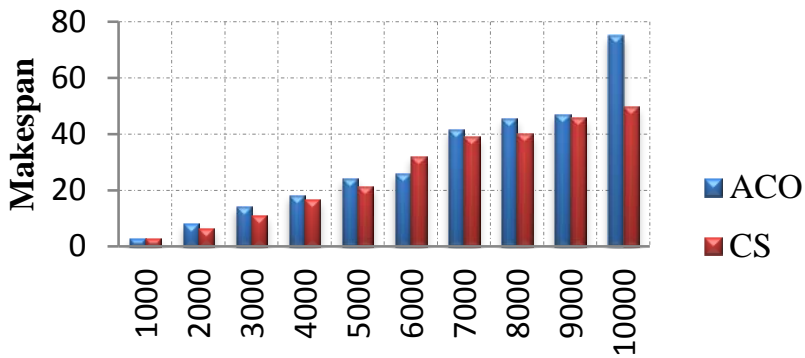

Cloudlet Lenght

Figure 4 Comparison of makespan for resource scheduling

The results are illustrated in Figure 4 reveal that the makespan of CS algorithm is minimum than ACO algorithm. So CS optimization algorithm has superior proficiency for resource scheduling in cloud computing.

Throughput

In generally, throughput is the rate of production or the rate in which something can be processed. In cloud computing, throughput means some tasks completed in a certain time period (Mustafa et al., 2015).

$$
\text { Throughput }=\sum_{\text {task }^{i}}\left(\text { Exe }_{\text {Time }}\right)
$$

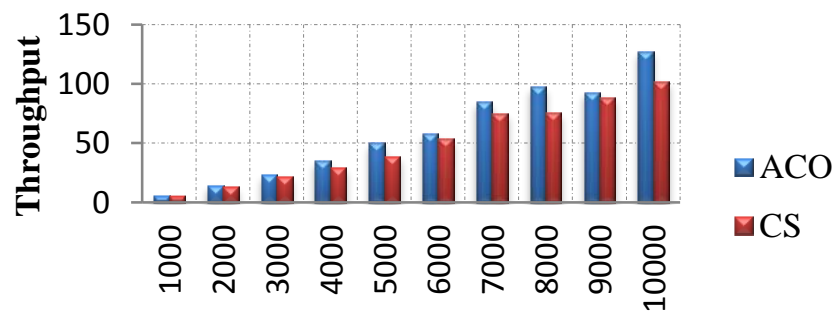

\section{Cloudlet Lenght}

Figure 5 Comparison of throughput for resource scheduling

The results as presented in Figure 5 demonstrate that the throughput of CS algorithm is lighter than the ACO algorithm. This implies that the proposed CS optimization algorithm has better performance rate for resource scheduling in cloud computing system.

\subsection{CONCLUSION}

In cloud computing environment, resource scheduling problem is one the main issues of research. The performance of resource scheduling algorithm in cloud computing is likely direct affecting the performance of the data center and overall cloud computing system. In this paper, we have used meta-heuristic CS algorithm and some aspects CS algorithm for resource scheduling in cloud computing. Our experiment shows that the proposed CS algorithm has outperformed the ACO meta-heuristic algorithm in terms of minimizing the response time, makespan and throughput for resource scheduling in IaaS cloud system.

In the future work, we will consider other quality matrices for optimization of resource scheduling in cloud computing. Furthermore, we would compare our proposed algorithm with other state-of-the-art meta-heuristic algorithms by considering the workload traces to find the most efficient and reliable algorithm that determines the optimal resource scheduling for cloud computing system. 
The authors are thankful to Universiti Teknologi Malaysia (UTM) for supporting this research through RUG grant Vote No.Q.J130000.2528.05H87.

\section{References}

Abdulhamid, S. M., Abd Latiff, M. S., Abdul-Salaam, G. \& Madni, S. H. H. (2016). Secure Scientific Applications Scheduling Technique for Cloud Computing Environment Using Global League Championship Algorithm. PLoS ONE, 11(7), e0158102.

Abdulhamid, S. M. \& Latiff, M. S. A. (2014). League Championship Algorithm Based Job Scheduling Scheme for Infrastructure as a Service Cloud. International Graduate Conference on Engineering, Science and Humanities 2014 Proceeding, 381-382.

Abdulhamid, S. M., Latiff, M. S. A. \& Bashir, M. B. (2014). Scheduling Techniques in on-demand Grid as a Service Cloud: A Review. Journal of Theoretical \& Applied Information Technology, 63(1), 10-19.

Abdulhamid, S. M., Latiff, M. S. A. \& Idris, I. (2015). Tasks Scheduling Technique Using League Championship Algorithm for Makespan Minimization in IaaS Cloud. ARPN Journal of Engineering and Applied Sciences, 9(12), 2528-2533.

Achar, R., Thilagam, P., Shwetha, D. \& Pooja, H. (2012). Optimal Scheduling of Computational Task in Cloud Using Virtual Machine Tree. Emerging Applications of Information Technology (EAIT), Third International Conference. IEEE, 143-146.

Armbrust, M., Fox, A., Griffith, R., Joseph, A. D., Katz, R., Konwinski, A., Lee, G., Patterson, D., Rabkin, A. \& Stoica, I. (2010). A View of Cloud Computing. Communications of the ACM, 53(4), 50-58.

Babukarthik, R., Raju, R. \& Dhavachelvan, P. (2013). Hybrid Algorithm for Job Scheduling: Combining The Benefits of ACO and Cuckoo Search. Advances in Computing and Information Technology. 177, 479-490.

Bashir, M. B., Abd Latiff, M. S., Ahmed, A. A., Yousif, A. \& Eltayeeb, M. E. (2013). Content-Based Information Retrieval Techniques Based on Grid Computing: A Review. IETE Technical Review, 30(3), 223-232.

Bibiks, K., Hu, F., Li, J.-P. \& Smith, A. (2015). Discrete Cuckoo Search for Resource Constrained Project Scheduling Problem. Computational Science and Engineering (CSE), 2015 IEEE 18th International Conference. IEEE, 240-245.

Dasgupta, P. \& Das, S. (2015). A Discrete Inter-Species Cuckoo Search for Flowshop Scheduling Problems. Computers \& Operations Research, 60, 111-120.

Foster, I., Zhao, Y., Raicu, I. \& Lu, S. Cloud Computing and Grid Computing 360-Degree Compared. Grid Computing Environments Workshop, 2008. IEEE, 1-10.

Gandomi, A. H., Yang, X.-S. \& Alavi, A. H. (2013). Cuckoo Search Algorithm: A Metaheuristic Approach To Solve Structural Optimization Problems. Engineering With Computers, 29(1), 17-35

Gharegozi, A. \& Jahani, R. 2013. A New Approach For Solving The Unit Commitment Problem by Cuckoo Search Algorithm. Indian Journal of Science and Technology, $6(9), 5235-5241$

Gunavathi, C. \& Premalatha, K. (2015). Cuckoo Search Optimisation for Feature Selection in Cancer Classification: A New Approach. International Journal of Data Mining and Bioinformatics, 13(3), 248-265.

Madni, S. H. H., Latiff, M. S. A. \& Coulibaly, Y. (2016). Resource Scheduling for Infrastructure As A Service (Iaas) In Cloud Computing: Challenges and Opportunities. Journal of Network and Computer Applications, 68, 173-200.

Madni, S. H. H., Latiff, M. S. A., Coulibaly, Y. \& Abdulhamid, S. I. M. (2016). An Appraisal of Meta-Heuristic Resource Allocation Techniques for IaaS Cloud. Indian Journal of Science and Technology, 9(4), 1-14.

Manvi, S. S. \& Krishna Shyam, G. (2014). Resource Management for Infrastructure as A Service (IaaS) in Cloud Computing: A Survey. Journal of Network and Computer Applications, 41, 424-440.

Mell, P. \& Grance, T. (2009). The NIST Definition of Cloud Computing. National Institute of Standards and Technology, 53, 50.

Mustafa, S., Nazir, B., Hayat, A. \& Madani, S. A. (2015). Resource Management in Cloud Computing: Taxonomy, Prospects, and Challenges. Computers \& Electrical Engineering, 47, 186-203.

Ouaarab, A., Ahiod, B. \& Yang, X.-S. (2014). Discrete Cuckoo Search Algorithm for the Travelling Salesman Problem. Neural Computing and Applications, 24(7), $1659-1669$

Raju, I. R. K., Varma, P. S., Sundari, M. R. \& Moses, G. J. (2016). Deadline Aware Two Stage Scheduling Algorithm in Cloud Computing. Indian Journal of Science and Technology, 9(4), 1-10.

Sait, S. M., Bala, A. \& El-Maleh, A. H. (2016). Cuckoo Search Based Resource Optimization of Datacenters. Applied Intelligence, 44(3), 489-506.

Tawfeek, M., El-Sisi, A., Keshk, A. E. \& Torkey, F. (2013). Cloud Task Scheduling Based on Ant Colony Optimization. 8th International Conference on Computer Engineering \& Systems (ICCES) Proceeding. IEEE, 64-69.

Wang, H., Wang, W., Sun, H., Cui, Z., Rahnamayan, S. \& Zeng, S. (2016). A New Cuckoo Search Algorithm with Hybrid Strategies for Flow Shop Scheduling Problems. Soft Computing, 1-11

Xu, X., Ji, Z., Yuan, F. \& Liu, X. (2014). A Novel Parallel Approach of Cuckoo Search using MapReduce. 2014 International Conference on Computer, Communications and Information Technology (CCIT 2014).

Yang, X.-S. \& Deb, S. (2009). Cuckoo search via Lévy flights. World Congress Nature \& Biologically Inspired Computing. IEEE, $210-214$.

Yang, X.-S. \& Deb, S. (2010). Engineering Optimisation by Cuckoo Search. International Journal of Mathematical Modelling and Numerical Optimisation, 1(4), 330343.

Yang, X.-S. \& Deb, S. (2013). Multiobjective Cuckoo Search for Design Optimization. Computers \& Operations Research, 40(6), 1616-1624.

Zendaoui, Z. \& Layeb, A. (2016). Adaptive Cuckoo Search Algorithm for the Bin Packing Problem. Modelling and Implementation of Complex Systems. 1, 107-120.

Zhu, Y. \& Liang, H. (2013). Research for the virtual Machine-Oriented Cloud Resource Scheduling Algorithm. 6th International Conference on Information Management, Innovation Management and Industrial Engineering (ICIII). IEEE, 133-136.

Zuo, L., Shu, L., Dong, S., Zhu, C. \& Hara, T. 2015. A Multi-Objective Optimization Scheduling Method Based on the Ant Colony Algorithm in Cloud Computing. Access, IEEE, 3, 2687-2699. 